\title{
Integrating Massage, Chiropractic, and Acupuncture in University Clinics: A Guided Student Observation
}

Alejandra A. Estrin Dashe PhD

College of Undergraduate Health Sciences, Northwestern Health Sciences University, Bloomington, MN, USA

Background: Several studies have reported on the health benefits of applying an integrated complementary health care model.

Purpose: This paper presents the results of pilot research focusing on the observations massage therapy students made about complementary health care education and integration during massage, chiropractic, and acupuncture treatments at two university clinics. Setting: Observations took place at Northwestern Health Sciences University's associated clinics that offered massage, chiropractic, and acupuncture. Research Design: Students directly observed how clinicians and interns educated their patients and integrated other forms of complementary health care into their practice. Participants: chiropractors, massage therapists, and acupuncturists, and their patients. All participants were English-speaking and 18-65 years old. Main Outcome Measures: Observations recorded by students in journals about education and integration during massage therapy, chiropractic, and acupuncture treatments were coded and counted.

Results: Qualitative observations showed that clinicians and interns educated patients to some degree, but the clinicians were less apt to integrate other modalities than the interns.

Conclusions: Observations support that professional integrity may limit clinicians in their ability to integrate multiple modalities of health care while treating patients. Since it is well established that integration of multiple health care modalities is beneficial to patient health, it is recommended that clinics assist their clinical staff in applying an integrative approach to their practice.

KEYWORDS: complementary and alternative medicine (CAM), health care, education, integration, massage, chiropractic, acupuncture, students

\section{INTRODUCTION}

\section{Background}

Patient education is instructed health information that empowers a patient with self-care methods to improve their own health. Providers of complementary and alternative medicine (CAM), such as massage, chiropractic, and acupuncture (MCA), improve health by emphasizing patient wellness and not illness while treating and educating patients ${ }^{(1)}$. For example, persons with chronic low back pain generally benefit from repeated acupuncture and massage treatments ${ }^{(1)}$, whereas persons with chronic arthritis ${ }^{(2)}$ or diabetes ${ }^{(3)}$ have improved health and lowered health care costs with self-care.

Integration of allopathic and complementary health care models into patient education and care have also improved patient health. Many allopathic medical clinics and hospitals have successfully integrated complementary health care practices into their health care philosophy ${ }^{(4-8)}$. Primarily, studies have shown that the key to successful integration was provider education $^{(4,5)}$. Secondarily, when providers knew about CAM integration benefits, they could educate patients about them. A partnership between the Oregon College of Oriental Medicine and Oregon Health and Science University showed that it was important for providers and patients to believe that acupuncture improved health in order for providers to recommend it and patients to choose it ${ }^{(7)}$. Beyond being helpful for patients, providers at the University of Pennsylvania ${ }^{(6)}$ and Melton Rutland Harborough Primary Care Group (England) ${ }^{(8)}$ believe that integration of CAM modalities could reduce health care costs.

All of these examples are important, but may make no difference to some health care providers because of personal opinion. CAM may not be recommended if the opinion is negative ${ }^{(7)}$. Providers may be biased against the technique because of "lack of evidence" stereotypes some people may have about $\mathrm{CAM}^{(9)}$. Additionally, economic competition between health care modalities could influence care, because one provider may not want lose money to another ${ }^{(10)}$.

CAM providers are not exempt from these limitations. Variation in CAM provider opinions appears to be the main reason why it has been difficult to integrate CAM modalities. Opinions include power (e.g., believing that one modality is superior to another), knowledge, and criticism about the effectiveness of other methods, or that the modalities are simply different and are the result of individual choices along a 
continuum of health care (e.g., ranging from natural remedy, wellness, accepted, and established modalities $)^{(8-12)}$. These limitations are especially problematic because providers separate each modality from the others as independent health care choices. Furthermore, this does not provide the best care for patients as evidenced by the relationships established between CAM and allopathic techniques ${ }^{(1-8)}$

Some health sciences universities are working on integrating complementary health care modalities and transmitting the benefits to the students and patients alike. CAM modality integration is the process of combining multiple health care disciplines (such as MCA) into one team that shares one paradigm: to heal and maintain wellness ${ }^{(10,13)}$. For example, at National University of Health Sciences (NUHS) in Lombard, Illinois, patients, clinicians, and interns reported that they would like to see more education and integration of the modalities ${ }^{(13)}$. Becoming comfortable with the knowledge may be a factor in the integration process. At least $50 \%$ of NUHS clinicians $(n=10)$ and interns $(n=89)$ said they were very familiar with massage therapy, chiropractic, acupuncture, and naturopathic treatment ${ }^{(13)}$

\section{Setting}

Northwestern Health Sciences University (NWH$\mathrm{SU}$ ) in Bloomington, $\mathrm{MN}$ is a CAM institution with four colleges: massage therapy, chiropractic, acupuncture, and an undergraduate program. NWHSU also has several teaching clinics that serve students in all colleges. The University has committed itself to improve the integration of MCA into an integrated complementary health care system ${ }^{(14)}$ by offering classes that address the commitment. One of the classes offered in the undergraduate program is a general Sociology class. As a way to fulfill the mission of the University, a requirement of Sociology students is to observe the culture of health care.

Based on the needs of the University to fulfill its mission and the general consensus in the academic literature that CAM modality integration is beneficial to patients, an objective of this pilot study is to begin to understand how education and integration of complementary health care is culturally transmitted between patients and providers. Also, a second objective is to show that observing patient-provider relationships (as an example of health care culture) can serve as an experiential learning model. It is hypothesized that providers educated patients about health care without regard to modality boundaries. The data presented are the Sociology students' observations, analysis, and recommendations for future student projects. The author also explores how this pilot study fits into a discussion about patient education and integrative relationships between MCA practices.

\section{METHODS}

\section{Sociology 1001}

Sociology 1001 is an introductory survey course about theory and methods of sociology. The course serves undergraduate students who want to obtain an associate degree in massage therapy or a bachelor degree in health sciences. The course description is as follows:

This course will guide students through an introductory study of various elements of human interaction. Historical elements related to the development of social theories will be reviewed. This course will review the concepts of social stratification, class, race and ethnicity, gender, culture, deviance as they relate to a study of Sociology. The topics of science, medicine and health care as they relate to a study of Sociology will provide a focus of this course. Fundamental concepts related to methodology of Sociology research will be addressed. We will examine all topics through the lens of the sociological perspective ${ }^{(15)}$, which is the ability to see and understand how general society works by observing the particular behaviors of people.

Ten students (three males, seven females) participated in the course. Eight were majoring in massage therapy, one was pre-chiropractic, and one was preacupuncture. The students received an explanation of the study on the first day of class.

\section{Participants}

Sociology 1001 students observed five appointments at any of three selected clinics: University Health Services (UHS), Pillsbury House (PH), and Winter Family Clinic (WFC). UHS and $\mathrm{PH}$ are free clinics that provide MCA with NWHSU clinical professors. UHS serves the NWHSU community and PH serves the local community in south Minneapolis (the Pillsbury neighborhood). WFC is a stand-alone clinic in south Minneapolis offering MCA. Anyone can receive care at the three clinics for low to no cost. Students registered with the clinics to observe appointments. Participants in this study were the patients seeking and providers of MCA practices at the three clinics. Appointments were selected by the receptionists at each clinic based on the participant parameters for patients: aged 18-65, English-speaking, and not a member of a protected class (minority, juvenile under the age of 18 , or pregnant). The receptionists were responsible for making sure the participant parameters were met. The participants were not approached before appointments. The study was explained to potential participants when they arrived in their treatment room.

\section{Human Subjects Protection}

This project was approved by the Northwestern Health Sciences University Institutional Review 
Board (NWHSU-IRB) on October 17, 2011 (AED95-09-11). Once approval was obtained, students were told to commence data collection. All participants in the study remained anonymous and no identifying information was recorded.

The research project in this course has intrinsic worth to each student seeking to develop an understanding of sociology and the methods by which sociological knowledge is generated. As a result, requiring participation of each student in the data collection was considered justified, and seeking an IRB for student participation in the course was not considered necessary. Further, the interdepartmental planning that set up the observational research and the training of students prior to their research activity presented an experience of extremely low-risk to everyone concerned - patients, providers, and students of the Sociology course. However, as a precaution in the future, subsequent studies involving student data collection will include an IRB for students participating in this course.

\section{Procedure}

At each appointment, the student observer introduced him/herself to the patients (people seeking treatment) and providers (clinicians and interns), explained the study, provided informed consent forms, secured informed consent, and quietly observed the patient-provider interaction. Students were asked to keep field notes, or write down their observations in a journal. Observations included only what they could see and hear during the appointment, because the IRB committee advised that it was safer for the patients and students to avoid communication. This meant that students could not collect data on the numbers of eligible participants, the number of participants who agreed or refused, or demographic data. However, students were allowed to speak to providers after patients were out of hearing range. Understandably, these limitations may have encouraged the Hawthorne Effect ${ }^{(16)}$ (observer influence on the social interaction) and made it difficult to tell if there was participant selection bias or demographic influence on the results. Putting these limitations in place was an important risk because they minimized patient risk and simplified the procedure students had to follow.

Data collection started on October 17, 2011 and continued through December 2, 2011. Data were analyzed in class starting on December 2, 2011 and at home with guidelines set by the professor. Students presented their findings as a poster or presentation as part of a final grade for the Sociology 1001 class on December 9, 2011. Students were also required to turn in their field notes, informed consent forms, and a copy of the poster or presentation in Microsoft Power Point (Microsoft Corporation, Redmond, WA) for grading and completing this report.

\section{Data Analysis \& Statistics}

The data from the students' field notes were coded (by the professor) to create the following variables: number of observations, number of providers (clinician and intern), number of patients, modality (chiropractic, massage, or acupuncture), location of services (UHS, $\mathrm{PH}$, or WFC), education, and modality integration. Number of observations refers to the number of times a student observed a treatment. Number of providers are the number of providers observed, and this was divided up by whether students observed clinicians (professional chiropractors, acupuncturists, or massage therapists who are teachers at the clinics and oversee intern education) and/or interns (students at NWHSU completing their clinical education). Number of patients refers to the number of patients observed. Modality refers to the type of treatment observed, either chiropractic, acupuncture, or massage. Education refers to the instances of observed health care education provided in the treatment. Modality integration refers to the instances of multiple observed treatments from more than one modality, during, in the past, or referred to for future health in the appointment. All quantitative data were recorded and analyzed using Microsoft Excel (Microsoft Corporation, Redmond, WA).

\section{RESULTS}

Of ten students in the Sociology 1001 course who performed observations, eight students turned in their field notes. The results reported and analyzed are based on those notes.

Referring to Tables 1 and 2, there were 30 total observations, where 31 patients and 41 providers were

TABle 1. Descriptive Statistics of Number of the Patient-Provider Interactions Observed

\begin{tabular}{lcccc} 
Clinics & Chiropractic & Acupuncture & Massage & Total \\
\hline University Health & 21 & 0 & 0 & 21 \\
Services & 7 & 1 & 1 & 9 \\
Pillsbury House & 28 & 1 & 1 & 30 \\
Total & & & \\
\hline
\end{tabular}

Table 2. Descriptive Statistics of the Number of Participants: Patients, Clinicians, and Interns Observed

\begin{tabular}{lcccc} 
Participants & Chiropractic & Acupuncture & Massage & Total \\
\hline Patients Observed & 29 & 1 & 1 & 31 \\
Clinicians Observed & 21 & 1 & 2 & 24 \\
Interns Observed & 17 & 0 & 0 & 17 \\
Total & 67 & 2 & 3 & 72 \\
\hline
\end{tabular}


observed. Students observed treatments in teaching clinics, and frequently saw interns and clinicians working together or, in one instance, there were two patients being treated together. Student observers mostly saw treatments at UHS. Only nine observations took place at $\mathrm{PH}$. No treatments were observed at WFC because it was more convenient for students to stay on campus and observe at UHS or relatively close by at $\mathrm{PH}$ (data not reported). The majority of treatments observed were chiropractic $(n=27$ at UHS and $\mathrm{PH}$, where $\mathrm{n}=21$ at UHS only).

Most of the students agreed that they consistently observed patients and providers communicating about health care education and that the providers did their job - they healed and educated their patients. However, upon analyzing the students' field notes, education communication was observed $27.5 \%$ of the time (11 out of 30 observations) and modality integration was observed $26.7 \%$ of the time ( 8 out of 30 observations). Further investigation and discussion with the Sociology students uncovered that they observed mostly interns educating, not clinicians. The students noted that it appeared that each clinician (chiropractors especially) wanted to treat their patient and move on to the next one. It is possible that the clinicians were moving quickly to accommodate several appointments. In comparison, interns may have spent more time with patients in order to learn how to become better clinicians, which may have given the impression of providing more education.

On the other hand, patients were observed asking their providers to educate them about other forms of health care. For example, one chiropractic patient asked their chiropractic intern about "therapeutic order"'(13), whether to get a massage or chiropractic treatment first. The intern suggested getting a massage first because it loosens up the joints for chiropractic. The patient was surprised by this response, but it is a standard practice. The surprise could have been a response to a cultural assumption that a chiropractor would encourage chiropractic first over massage. In fact, it was observed that some of the clinicians encouraged treatment from their own professions first. There were two instances of professional preference observed, detailed in the next two paragraphs.

In one case, a patient asked about seeking a second opinion from an orthopedic surgeon. The chiropractor gave their personal experience with this form of allopathic medicine. The student observer thought that the chiropractor was "pushy" about his opinion regarding getting chiropractic treatment over orthopedic surgery, but in the end said to the patient that they could do what they wanted.

In another observation, an acupuncturist suggested to a patient to wait on massage therapy so that the focus of the treatment would be on acupuncture first. It seemed to the students observing that the clinician did not wish for their patients to engage in another modality until their own modality exhausted all health care possibilities, and clinicians tried to talk patients out of going to other providers. These instances, while few, might suggest that clinicians show some discomfort or resistance to integration.

The observations of the interns provided a different scenario. During one observation, a chiropractic intern asked the student observer what they would do as a massage therapist to help the patient. This put the student observer in an uncomfortable social situation. It was clear to the observing student that the intern felt comfortable asking the student observer their opinion. However, the student observer could not say anything, even if they wanted, because they were not allowed to treat patients and only observe as stipulated by the IRB.

Finally, the Sociology students noted that there was a difference in how patients were seen by providers. Observations at UHS showed that clinicians were modeling independent practices for their interns because of how the appointments were arranged - in rooms big enough for one provider and one patient. Observations at $\mathrm{PH}$ showed that integration worked because the appointments were arranged in a larger room with space for several providers to work on one patient. The students noted that education and integration would have been easier if treatments could have been done in larger rooms or with floating massage therapists, chiropractors, and acupuncturists around during each appointment.

\section{DISCUSSION}

\section{Patient-Provider Interactions and CAM Integration}

The students' observations generally show that interns and clinicians experience different patientprovider interactions. The interns in this pilot study provided education and promoted integration of multiple MCA modalities whereas the clinicians were not as active. Such findings partially concur with recently published data: NUHS clinicians and interns were familiar with, interested in, and actively promoted education and integration of more than one MCA modality into their practice ${ }^{(13)}$. Possible reasons why the NWHSU interns seem more active in education and integration than clinicians are presented in this discussion.

Interns are invested in their own education. They are learning multiple modalities at once, particularly while in a clinic setting. This is especially true at $\mathrm{PH}$, where several modalities are used on the same patient in the same appointment. Interns at other universities have shown this pattern as well. Acupuncture interns at the Oregon College of Oriental Medicine stated that they learned more about conventional medicine while interning as acupuncturists at a conventional medical 
clinic $^{(7)}$. Interns are also under the educational direction of clinicians. They may feel some academic freedom in not knowing what to do in every instance. Interns can call upon a second provider to assist in patient treatment, which may explain why one chiropractic intern asked the student observer to assist him/her with their patient.

On the other hand, MCA clinicians may not feel like they have the same flexibility as the interns to recommend or support other forms of health care because of paradigmatic constraints. They may be more accepting of paradigms from their chosen professions and, possibly, are not as accepting of others because that is how they were trained ${ }^{(10)}$. There also may be a perceived superiority of MCA practices over others because of pride and need to dominate the market. Perceived superiority may also encourage "use of closure or exclusive strategies aimed to limit or regulate the entering of competitors into the market'(10). In other words, there are some economic incentives to promoting MCA practices over others. The result is a power structure that is difficult to disengage: that one's own MCA practice is more important or better than others. In turn, the health care market is dominated by one type of health care over others and is a disintegrated system.

MCA clinicians may promote their own practice, but may also involuntarily disempower their patients. A resulting effect is less patient agency to make informed health care choices when only one type of care is provided. In the instances observed where the clinician suggested acupuncture over massage or chiropractic over orthopedic surgery, the patients' agencies in their health care were muted by the clinicians' opinions. These observations show that it may be important to teach providers how to promote health care integration without offending MCA practice integrity, as well as why integration is beneficial to patients seeking wellness.

One way to begin the process of making changes in how education and integration are applied in clinical settings is to model the behavior for the interns. Interns know that they should provide education to their patients. Yet when interns observe clinicians in action, it is important that the clinicians are able to show that they believe in what they do and what other MCA providers do, too.

The Sociology students also suggested a few ways to encourage integration. One way would be to improve massage education techniques for chiropractic students so that it is up to the level that massage therapists learn them. Another way to support an integrated model would be to provide a floating MCA provider in case a recommendation is needed. Teaching chiropractors more massage and having extra staff at a clinic would relieve a provider from having to wonder what another provider would do.

Finally, clinicians need training opportunities to become comfortable with an integrated model.
Educating providers about other health modalities is important for delivering good health care, even if most providers already know about them ${ }^{(13)}$. It is also equally important to educate providers when to recommend more than one form of health care without professionally offending other providers. This form of training may be necessary to culturally incorporate complementary health care modality integration into professional MCA practice.

\section{Learning from Students: Limitations and Future Study}

One of the limitations to this pilot study is that the Sociology students' presence in the treatment room influenced the behaviors of the patients and providers and, therefore, their observations, which is also known as the Hawthorne Effect ${ }^{(16)}$. This psychological variable should be eliminated from future studies. Future studies would focus on collecting data behind a video camera in another room, so that patients and providers are not aware that they are being observed (but have given consent ahead of time).

Sociology students also wanted to know more about the patient-provider interaction beyond just observing without interaction. The next group of students should interview patients and providers directly before and/or after the treatment to get more specific data about demographics, how the patients and providers communicate, and details on integration of health care modalities. Interviews would need to be recorded and transcribed to provide more information about patient-provider interactions and CAM modality integration.

The study was a pilot study. As such, the entire body of literature on educational intervention about the integration of multiple CAM modalities was not presented. Future studies will include a greater literature review. Also, only three clinics were associated with this study and the study was limited the participants to English-speaking patients only. As a result, the study had a small sample size and broad conclusions. The next round of data collection would be expanded to include all health care clinics on the NWHSU campus and non-English speaking patients, so that sample size and data collection are increased to confirm the broad conclusions made in this study.

\section{CONFLICT OF INTEREST NOTIFICATION}

The authors declare there are no conflicts of interest.

\section{COPYRIGHT}

Published under the CreativeCommons AttributionNonCommercial-NoDerivs 3.0 License. 


\section{REFERENCES}

1. Cherkin D, Eisenberg D, Sherman K, et al. Randomized trial comparing traditional Chinese medical acupuncture, therapeutic massage, and self-care education for chronic low back pain. Arch Intern Med. 2001;161(8):1081-1088.

2. Lorig K, Mazonson P, Holman H. Evidence suggesting that health education for self-management in patients with chronic arthritis has sustained health benefits while reducing health care costs. Arthritis Rheum. 1993;36(4):439-446.

3. Renders C, Valk G, Griffin S, et al. Interventions to improve the management of diabetes in primary care, outpatient, and community settings. Diabetes Care. 2001;24(10):1821-1833.

4. Giordano J, Boatwright D, Stapleton S, et al. Blending the boundaries: steps toward an integration of complementary and alternative medicine into mainstream practice. $J$ Altern Complement Med. 2002;8(6):897-906.

5. Highfield E, McLellan M, Kemper K, et al. Integration of complementary and alternative medicine in a major pediatric teaching hospital: an initial overview. J Altern Complement Med. 2005;11(2):373-380.

6. Mao J, Kapur R. Acupuncture in primary care. Primary Care. 2010;31(1):105-117.

7. $\mathrm{Wu} \mathrm{J}, \mathrm{Lu} \mathrm{Z}$, Hayes M, et al. Integration of acupuncture into family medicine teaching clinics. J Altern Complement Med. 2009;15(9):1015-1019.

8. Lipman L, Dale J, MacPherson H. Attitudes of GPs towards the provision of acupuncture on the NHS. Complement Ther Med. 2003;11(2):110-114.

9. Hehir L, Williams S. A survey of health professionals' views about integration of Traditional Chinese Medicine (TCM) Acupuncture into NHS Scotland. European Journal of Integrative Medicine. 2011;3(4):e1-e7. Published online December 14, 2011.
10. Adams J, Hollenberg D, Lui C, et al. Contextualizing integration: a critical social science approach to integrative health care. J Manipulative Physiol Ther. 2009;32(9):792-798.

11. Hollenberg D, Tsasis P, Kelley N. CAM in Canadian hospitals: the new frontier? $J$ Compliment Integrative Med. 2011;8(1):Article 20. Published online June 2011.

12. Robinson A, Chesters J, Cooper S. Beyond a generic complementary and alternative medicine: the holistic health care-conventional medicine continuum. Journal of Evidenced-based Complementary and Alternative Medicine. 2009;14(s3):153-163.

13. Cohen E, Cambron J, Sheil R. Measuring the development of integrative care communiation patterns and cross-disciplinary knowledge at a university clinic: a baseline cross-sectional study. J Manipulative Physiol Ther. 2009;32(9):758-764.

14. NWHSU. Mission, Vision and Guiding Principles. Northwestern Health Sciences University. 2011. Available from: http://nwhealth.edu/get-to-know/mission-vision-principles/. Accessed December 21, 2011.

15. Berger P. Invitation to Sociology. A Humanistic Perspective. New York: Anchor Books; 1963. p. 1-24.

16. Wickström G, Bendix T. The "Hawthorne effect" — what did the original Hawthorne studies actually show? Scand J Work, Environ Health. 2000;26(4):363-367.

Corresponding author: Alejandra A. Estrin Dashe, $\mathrm{PhD}$, College of Undergraduate Health Sciences, Northwestern Health Sciences University, 2501 W. $84^{\text {th }}$ St., Bloomington, MN, 55431 USA

E-mail: adashe@nwhealth.edu 